\title{
Scaling of $B_{K}$ for $\mathbf{2 + 1}$ flavour domain wall fermions from $24^{3}$ and $32^{3} \times 64$ lattices
}

\author{
Chris Kelly* \\ The University of Edinburgh \\ School of Physics \\ James Clerk Maxwell Building, King's Buildings \\ Mayfield Road \\ Edinburgh EH9 3JZ, UK \\ E-mail: christopher.kellyeed.ac.uk
}

\section{For the RBC and UKQCD collaborations}

We present preliminary results from a $32^{3} \times 64$ lattice calculation of the neutral kaon mixing factor $B_{K}$ using $2+1$ flavours of Domain Wall fermions, focussing on the chiral extrapolation and non-perturbative renormalisation (NPR) of this quantity and comparing to our previous results obtained from a $24^{3} \times 64$ lattice.

These simulations were performed using the Iwasaki gauge action at two different lattice spacings: $a^{-1}=1.729(28) \mathrm{GeV}$ for the $24^{3} \times 64$ calculation and $a^{-1}=2.42(4) \frac{0.47}{r_{0}(\mathrm{fm})} \mathrm{GeV}$ for the $32^{3} \times 64$, maintaining an approximately fixed physical volume of $(2.7 \mathrm{fm})^{3}$.

In the discussion of the chiral extrapolation to the physical quark masses, we compare the fits to an $S U$ (2) partially-quenched chiral fit form for which the kaon is coupled into the theory as a heavy external particle. As the $32^{3} \times 64$ results are preliminary at the time of writing we do not present a result for the chiral extrapolation on this data set. Results for the $24^{3} \times 64$ lattice calculation have been published [1].

For the NPR we compare the stages of the calculation of the non-perturbative renormalisation constant $Z_{B_{K}}$ using the Rome-Southampton regularisation-invariant momentum scheme (RI/MOM), where for the recent $32^{3} \times 64$ calculation we have used a lattice-volume source for the calculation of the propagators, an approach which offers a substantial gain over the point source approach used for the $24^{3} \times 64$ calculation due to the volume averaging of the bilinear and four-quark vertex locations.

The XXVI International Symposium on Lattice Field Theory

July 14 - 19, 2008

Williamsburg, Virginia, USA

\footnotetext{
* Speaker.
} 


\section{Introduction}

Neutral kaon mixing can be parameterised through an amplitude

$$
A\left(K^{0} \rightarrow \bar{K}^{0}\right)=\frac{G_{F}}{2} \sum_{i} V_{\mathrm{CKM}}^{i} C_{i}(\mu)\left\langle K^{0}|Q(\mu)| \bar{K}^{0}\right\rangle,
$$

for which $V_{\mathrm{CKM}}^{i}$ are CKM-matrix elements, $C_{i}$ are scheme-dependent perturbative factors incorporating contributions from physics at high energy scales and the matrix element $Q$ is a nonperturbative scheme dependent quantity containing the QCD interactions at the hadronic energy scale. The matrix element is parameterised by a quantity $B_{K}$, defined as

$$
B_{K} \equiv \frac{\left\langle K^{0}\left|\mathscr{O}_{V V+A A}\right| \bar{K}^{0}\right\rangle}{\frac{8}{3} f_{K}^{2} M_{K}^{2}},
$$

where the four-quark operator $\mathscr{O}_{V V+A A}$ is defined as

$$
\mathscr{O}_{V V+A A} \equiv\left(\bar{s} \gamma_{\mu} d\right)\left(\bar{s} \gamma_{\mu} d\right)+\left(\bar{s} \gamma_{5} \gamma_{\mu} d\right)\left(\bar{s} \gamma_{5} \gamma_{\mu} d\right)
$$

$B_{K}$ can be perturbatively related to the measure of indirect CP-violation in the kaon sector $\varepsilon_{K}$ via the operator product expansion [5]. This relation contains the CKM matrix phase $\delta$ which allows for direct CP-violation within the Standard Model. $\varepsilon_{K}$ can be measured experimentally through the ratio

$$
\varepsilon_{K}=\frac{K_{L} \rightarrow(\pi \pi)_{I=0}}{K_{S} \rightarrow(\pi \pi)_{I=0}}
$$

and is known to a high degree of precision, thus by measuring $B_{K}$ we can constrain the value of $\delta$. As a non-perturbative pure QCD quantity, $B_{K}$ is ideally suited for calculation by lattice methods.

In these proceedings we discuss the calculation of $B_{K}$ upon two Domain Wall fermion ensembles of differing lattice spacings but approximately equal physical volumes. Our simulations were performed at lattice volumes of $24^{3} \times 64$ and $32^{3} \times 64$ with $L_{s}=16$ using the Iwasaki gauge action at $\beta=2.13$ and 2.25 respectively, giving physical lattice spacings of $a^{-1}=1.729(28) \mathrm{GeV}$ and $a^{-1}=2.42(4) \mathrm{GeV}$ and physical volumes of $(2.74 \mathrm{fm})^{3}$ and $\sim(2.61 \mathrm{fm})^{3}$. Here the $32^{3} \times 64$ lattice spacing is given assuming $r_{0}=0.47 \mathrm{fm}$.

The layout of these proceedings is as follows: we briefly discuss and compare the approaches to the measurement of the $B_{K}$ (unrenormalised) matrix element upon our two ensembles and follow by an in-depth discussion of our chiral extrapolation and non-perturbative renormalisation techniques. The results for the $32^{3} \times 64$ simulation are preliminary and from a partial dataset, thus we refrain from quoting final values for the calculations.

\section{Lattice determination of $B_{K}$}

Upon our $24^{3} \times 64$ ensemble we measured the $B_{K}$ matrix element of equation 1.2 using propagators calculated from two temporally separated wall sources in the Coulomb gauge. We calculated each propagator using both periodic (p) and antiperiodic (a) boundary conditions, which when combined in summation $(\mathrm{p}+\mathrm{a})$ forces the cancellation of the combined propagator at the lattice boundary, preventing the unwanted round-the-world pion contributions to the matrix element. 


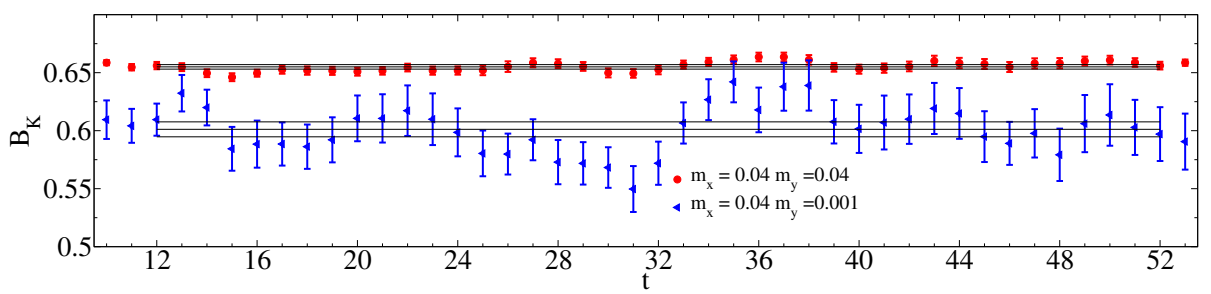

(a)

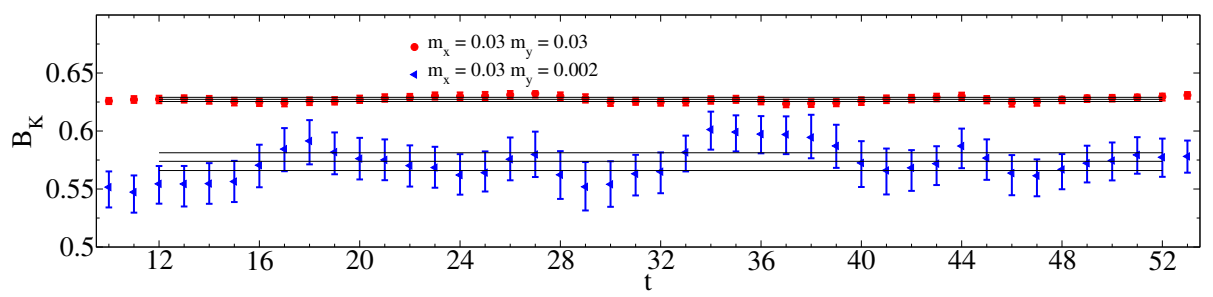

(b)

Figure 1: Example plateaux from our $24^{3} \times 64$ (upper) and $32^{3} \times 64$ (lower) ensembles for the heavy-heavy and heavy-light states.

The sources were placed at large temporal separations, specifically $t=5$ and 59 , in order to provide a large plateau length. Details of this calculation can be found in ref. [2].

For the $32^{3} \times 64$ measurements we used a single Coulomb gauge-fixed wall source located upon the lattice boundary $t=0$, again calculating the propagator using both periodic and antiperiodic boundary conditions. As the source is located on the boundary, the p+a combination gives a purely forwards propagating quark moving away from the boundary at $t=0$ and the $\mathrm{p}$-a combination gives a backwards propagating quark propagating away from the boundary at $t=64$. By using these propagators for the matrix element we are able to maximise the plateau length at one-half of the cost of the previous approach. This enables more frequent measurements to be made.

In figure 1 we show example plateaux from both of our ensembles.

\section{Chiral Extrapolation}

We calculated the raw $B_{K}$ matrix element using a partially-quenched data set to maximise the use of our ensembles. In order to find the physical (unrenormalised) value of $B_{K}$ we must extrapolate to the physical quark mass. We use $S U(2)$ partially-quenched chiral perturbation theory (PQ $\chi \mathrm{PT})$ to calculate the fit forms, in which the kaon is coupled into the the theory at lowest order in the relativistic expansion. This approach has a direct connection to heavy-meson chiral perturbation theory. The chiral fit form

$$
B_{K}=B_{K}^{0}\left[1+\frac{2 B\left(m_{d}+m_{\mathrm{res}}\right) c_{0}}{f^{2}}+\frac{2 B\left(m_{y}+m_{\mathrm{res}}\right) c_{1}}{f^{2}}-\frac{2 B\left(m_{y}+m_{\mathrm{res}}\right)}{32 \pi^{2} f^{2}} \log \left(\frac{2 B\left(m_{y}+m_{\mathrm{res}}\right)}{\Lambda_{\chi}^{2}}\right)\right]
$$

contains four low-energy constants $B, f, c_{0}$ and $c_{1}$ and $B_{K}^{0}$ defined as the chiral limit of the $B_{K}$ matrix element. In order to constrain this fit we perform pure $S U(2)$ partially-quenched chiral fits 


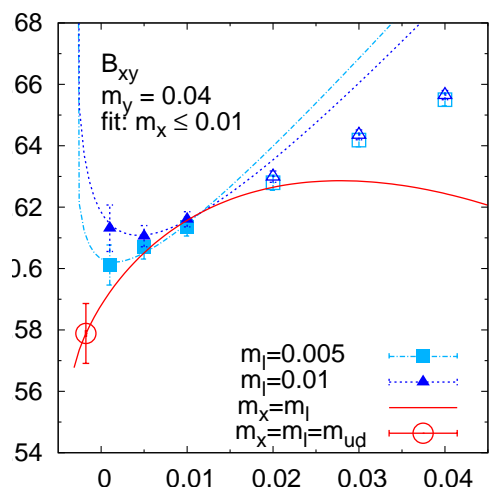

(a)

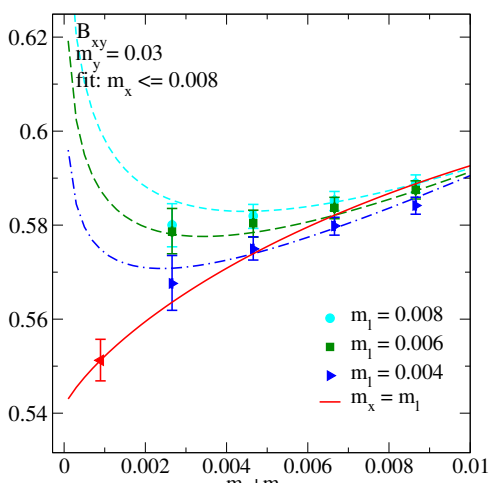

(b)

Figure 2: PQ $\chi \mathrm{PT}$ fits to $B_{K}$ data from our $24^{3} \times 64$ (left) and $32^{3} \times 64$ (right) ensembles.

simultaneously to $M_{\pi}$ and $f_{\pi}$ to determine $B$ and $f$. For further details of our methods we refer the

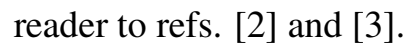

Figure 2 shows examples of $S U(2)$ PQ $\chi$ PT fits to $B_{K}$ upon both of our ensembles. The results for the $24^{3} \times 64$ ensembles have been published [2]. The data is first fitted to the above form and extrapolated along the unitary line (red) to the physical point.

\section{Non-perturbative Renormalisation}

$B_{K}$ is a scale and scheme dependent quantity and as such must be renormalised to the conventional $\overline{\mathrm{MS}}$ scheme at $2 \mathrm{GeV}$. We match via the Rome-Southampton regularisation-invariant momentum scheme (RI/MOM). This approach uses only non-perturbative lattice data and continuum perturbation theory, avoiding the convergence issues and prescription dependent ambiguities present in current lattice (DWF) perturbative calculations as discussed in ref. [1].

This method is as follows. The four-quark $\mathscr{O}_{V V+A A}$ vertex is calculated in Landau gauge and the external quark legs are amputated using the (unrenormalised) ensemble-averaged quark propagators. The renormalisation constant for the $\mathscr{O}_{V V+A A}$ operator $Z_{V V+A A}(\mu)$ is defined as the factor that takes the amputated vertex to its tree level value at the scale $\mu^{2}=p^{2}$ where $p$ is the quark momentum:

$$
\frac{Z_{V V+A A}(\mu)}{Z_{q}^{2}} \Lambda_{\mathscr{O}_{V V+A A}}(\mu)=\Theta_{\mathscr{O}_{V V+A A}}^{\mathrm{tree}}
$$

Here $\Lambda_{\mathscr{O}_{V V+A A}}$ is the amputated vertex, $\Theta_{\mathscr{O}_{V V+A A}}^{\text {tree }}$ is the tree level amplitude and the factors of $Z_{q}$ are included due to the use of unrenormalised propagators in the amputation.

The renormalisation constant for $B_{K}, Z_{B_{K}}^{\mathrm{RI} / \mathrm{MOM}}$, is obtained from this by dividing by the appropriate factors for the renormalisation of the axial-current bilinears used to obtain the denominator of eqn. 1.2. As the Domain Wall fermion Lagrangian is approximately chirally-symmetric, in principle either the vector or axial renormalisation constants could be used:

$$
\Lambda_{A}=\Lambda_{V}=\frac{Z_{q}}{Z_{A}}=\frac{Z_{q}}{Z_{V}}
$$




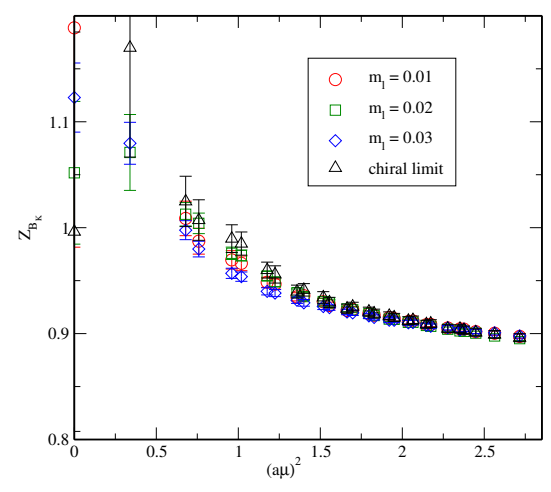

(a) $Z_{B_{K}}$ as a function of the momentum scale $\mu$ for 3 unitary quark masses.

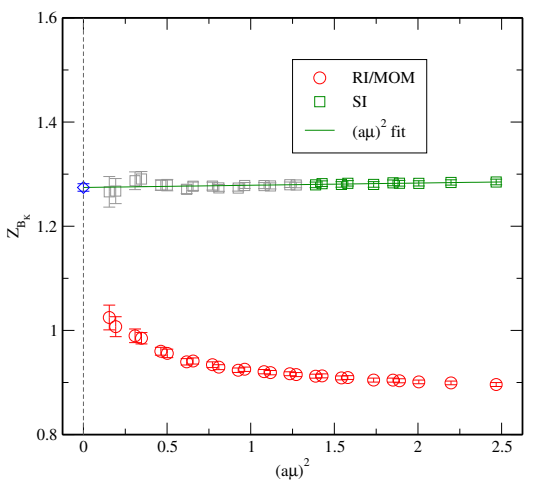

(b) $Z_{B_{K}}$ before (RI/MOM) and after (SI) the perturbative running is divided out, showing the quadratic fit in $\mu$ used to remove lattice artefacts.

Figure 3: Plots showing two stages of the calculation of $Z_{B_{K}}$ on our $16^{3} \times 32$ ensembles.

providing the renormalisation scale is much higher than the scale at which QCD spontaneously breaks the chiral symmetry. Here $\Lambda_{A}$ and $\Lambda_{V}$ are amputated bilinear vertices and the bilinear renormalisation condition is the same as that of the four-quark vertex. In practise we find that $\Lambda_{A}$ and $\Lambda_{V}$ differ at order $2 \%$ even at high momenta. This is due to our use of an exceptional momentum configuration for our bilinear and four-quark vertices, defined as a configuration in which there is a net zero sum of momentum at the vertex if all external momenta are directed into the graph. These configurations reduce the suppression of low-energy chiral symmetry breaking subgraphs allowing the effects of the low-energy chiral symmetry breaking to be felt even if the incoming momenta are hard [1]. We use the average of $\Lambda_{A}$ and $\Lambda_{V}$ to calculate $\frac{Z_{q}}{Z_{A}}$.

Once we have calculated $Z_{B_{K}}^{\mathrm{RI} / \mathrm{MOM}}$ for a range of momenta and unitary quark masses we perform a linear chiral extrapolation to $-m_{\text {res }}$, the residual mass of the Domain Wall fermions. We then divide out the perturbative running of this quantity, leaving a value $\tilde{Z}_{B_{K}}^{S I}$ which is scale-invariant up to lattice artefacts of order $(a \mu)^{2}$ that can be removed by fitting $\tilde{Z}_{B_{K}}^{S I}$ to the quadratic form

$$
\tilde{Z}_{B_{K}}^{S I}(\mu)=Z_{B_{K}}^{S I}+B(a \mu)^{2} .
$$

We then reapply the perturbative running to the scale invariant quantity $Z_{B_{K}}^{S I}$ and run to the conventional scale of $\mu=2 \mathrm{GeV}$. This can then be perturbatively converted into the $\overline{\mathrm{MS}}$ scheme.

Our $24^{3} \times 64$ ensembles were generated with the Iwasaki gauge action using the same $\beta=2.13$ as our older $16^{3} \times 32$ lattices, allowing us to reuse the renormalisation coefficients calculated on these older ensembles. Here we briefly summarise the calculation; further details can be found in ref. [1]. We used point-source propagators, forming the four-quark and bilinear vertices at the source location. This method allows us to access all quark momenta using a single propagator but is rather susceptible to statistical fluctuations in the gauge field as the vertices are formed on only a single lattice site. Figure 3 shows $Z_{B_{K}}$ as a function of the momentum scale, its chiral extrapolation, the removal of the perturbative running and the subsequent extrapolation to $\mu=0$. We quote the 


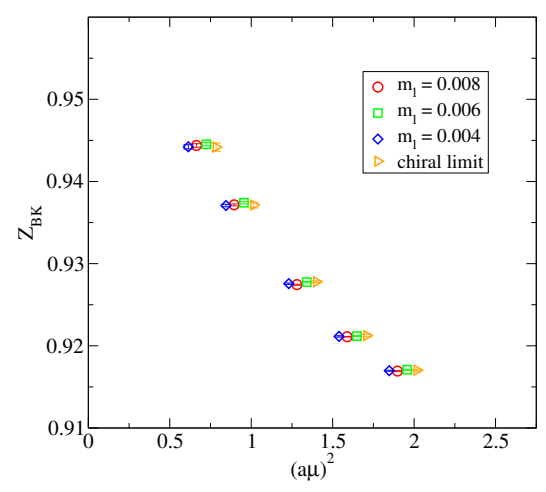

(a) $Z_{B_{K}}$ as a function of the momentum scale $\mu$ for 3 unitary quark masses. Points for each of the $5 p^{2}$ values have been shifted for clarity.

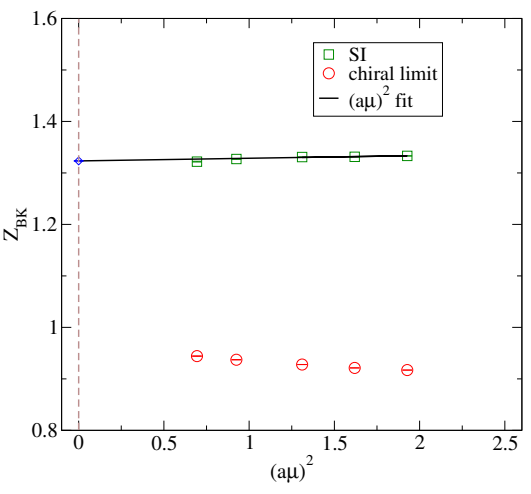

(b) $Z_{B_{K}}$ before (RI/MOM) and after (SI) the perturbative running is divided out, showing the quadratic fit in $\mu$ used to remove lattice artefacts.

Figure 4: Plots showing two stages of the calculation of $Z_{B_{K}}$ on our $32^{3} \times 64$ ensembles.

value obtained for $Z_{B_{K}}$ in the $\overline{\mathrm{MS}}$ scheme at $2 \mathrm{GeV}$ :

$$
Z_{B K}^{\overline{\mathrm{MS}}}(2 \mathrm{GeV})=0.9276 \pm 0.0052 \text { (stat) } \pm 0.0220 \text { (sys). }
$$

For our $32^{3} \times 64$ ensembles we have used lattice volume sources, forming the vertex at the propagator sink and averaging over all lattice sites. This method has the advantage of a full volume average over lattice sites but requires a separate inversion of the propagator for each desired quark momentum. Figure $\emptyset$ shows $Z_{B_{K}}$ as a function of the momentum scale calculated using 5 pairs of momenta with equal $p^{2}$ for three unitary quarks calculated on only 20 gauge configurations per quark; we found that the statistical fluctuations of the data calculated using the volume source approach were so small that using more configurations is unnecessary. From these plots it is evident that the volume source approach offers a significant advantage over the traditional point source approach, giving vastly smaller statistical errors for a lower cost.

From eqn. 4.4 it is evident that the calculation of $Z_{B_{K}}$ is dominated by systematic errors, the largest contribution being due to our use of exceptional momentum configurations. For our $32^{3} \times 64$ calculations we chose our pairs of momenta such that they can be combined to form a non-exceptional momentum configuration, allowing us to compare the two approaches. Figure 5 demonstrates that using a non-exceptional momentum configuration removes the ambiguity in the calculation of $\frac{Z_{q}}{Z_{A}}$. Unfortunately to our knowledge there are currently no appropriate calculations of the perturbative running of $Z_{B_{K}}$ using these non-exceptional momentum kinematics, although such calculations have been performed for for other quantities such as $Z_{m}$ [ $[$ ].

\section{Conclusions and Outlook}

For our $24^{3} \times 64$ ensemble we calculated a final value for $B_{K}$ in the $\overline{\mathrm{MS}}$ scheme at $2 \mathrm{GeV}$ :

$$
B_{K}^{\overline{\mathrm{MS}}}(2 \mathrm{GeV})=0.524(10)_{\mathrm{stat}}(13)_{\mathrm{ren}}(25)_{\mathrm{sys}}
$$




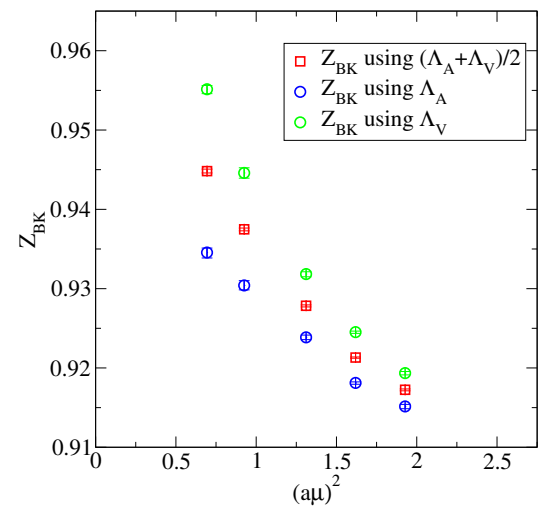

(a)

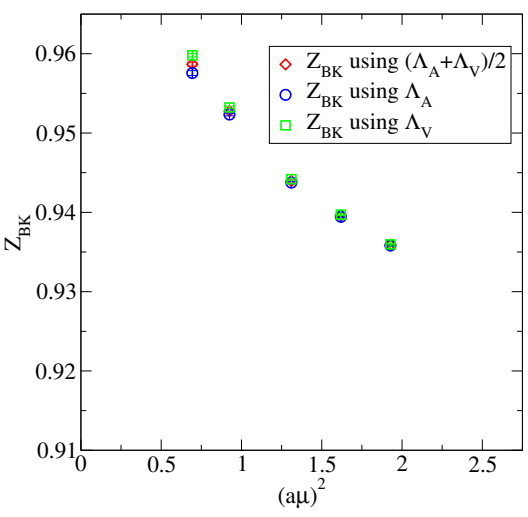

(b)

Figure 5: Plots showing $Z_{B_{K}}$ as a function of $(a \mu)^{2}$ calculated using exceptional (left) and non-exceptional (right) configurations.

where the statistical ('stat') error combines that of the NPR and $B_{K}$ calculations and the systematic ('sys') error contains all systematic contributions apart from those of the non-perturbative renormalisation ('ren'). The largest contribution to the 5\% systematic error arises from the lack of an extrapolation in lattice spacing $(4 \%)$. Our new $32^{3} \times 64$ data will allow us to perform this extrapolation, reducing the systematic error to an expected $\sim 3 \%$.

This precision calculation will allow for the exploitation of the precisely determined measure of indirect CP-violation $\varepsilon_{K}$ to constrain the CKM phase $\delta$. By combining this with unitarity triangle constraints it may be possible to determine whether $\delta$, the only Standard Model parameter capable of describing CP-violation, is sufficient to describe the experimental data.

\section{Acknowledgements}

I am thankful to all the members of the UKQCD and RBC-Collaborations. The computations were performed on the QCDOC machines at the University of Edinburgh and Columbia University, the US DOE and RBRC facilities at the Brookhaven National Laboratory and the BG/P machines at Argonne National Laboratory. The author was supported by the UK STFC.

\section{References}

[1] RBC and UKQCD collaborations, Y. Aoki et al., Non-perturbative renormalization of quark bilinear operators and $B_{K}$ using domain wall fermions, arXiv:0712.1061 [hep-lat].

[2] RBC and UKQCD collaborations, C. Allton et al., Physical Results from 2+1 Flavor Domain Wall $Q C D$ and $S U(2)$ Chiral Perturbation Theory, arXiv:0804.0473 [hep-lat].

[3] RBC and UKQCD collaborations, E. Scholz, Physical results from 2+1 flavor Domain Wall QCD, PoS(LATTICE 2008)095.

[4] RBC and UKQCD collaborations, Y. Aoki, Quark mass renormalization with non-exceptional momenta, PoS(LATTICE 2008)222.

[5] A. J. Buras, Weak Hamiltonian, CP violation and rare decays, arXiv:hep-ph/9806471. 